\title{
Relativistic Hirshfeld atom refinement of organo-gold(I) and organo-mercury(II) compounds
}

\author{
Sylwia Pawlędzioa, Maura Malinska ${ }^{a}$, Florian Kleemiss ${ }^{b}$, Simon Grabowsky ${ }^{b}$, and Krzysztof Woźniak \\ ${ }^{a}$ Biological and Chemical Research Centre, Department of Chemistry, University of Warsaw, Żwirki i Wigury 101, 02-089 Warsaw, \\ Poland \\ ${ }^{b}$ University of Bern, Department of Chemistry and Biochemistry, Freiestrasse 3, 3012 Bern, Switzerland \\ spawledzio@uw.edu.pl
}

Relativistic effects in chemistry manifest themselves in many ways and influence various physical and chemical properties of materials. The well-known of them is the yellow color of gold or the high voltage of the lead-acid car battery [1,2]. Therefore, a description of these effects is of great importance for a better understanding of the chemistry of heavy atoms.

A perspective method is quantum crystallography that relies on the high-resolution and high-quality XRD data to describe crystal structure in unprecedented detail [3,4]. Intensities of the diffracted beam are affected not only by relativistic effects but also by absorption [5], anharmonic motion [6], anomalous dispersion [7], and many other effects which highly influence electron density distribution in the crystal and, in consequence, derived properties.

We collected the data sets for the chloro(triphenylphosphine)gold(I) and di(triphenylphosphine)mercury(II) nitrate using Mo X-ray source at 100K, where the data sets for (3-(4-chlorophenyl)-3-oxoprop-1-yn-1-yl)(triphenylphosphine)gold(I) and chloro (dimethylsulfide) gold(I) were collected using synchrotron radiation at $80 \mathrm{~K}$.

Here, we present the results of relativistic Hirshfeld atom refinements ${ }^{8}$ carried out as implemented in NoSpherA $2^{9}$ for high-resolution X-ray diffraction data sets. The outcome of DFT-based refinements with the nonrelativistic and quasi-relativistic approaches will be compared, including analysis of the influence of disorder on relativistic effects, description of aurophilic interactions, and the nature of the $\mathrm{Me}-\mathrm{X}$ bonds in $\mathrm{Au}$ and $\mathrm{Hg}$ crystals.

1. Pyykko, P. (1988). Chemical Reviews, 88, 563-594.

2. Yatsimirskii, K. B. (1995) Theoretical and Experimental Chemistry, 31, 153-168.

3. L. J. Farrugia, C. Evans, D. Lentz and M. Roemer, Journal of the American Chemical Society, 2009, 131, 1251-1268.

4. T. S. Koritsanszky and P. Coppens, Chem. Rev., 2001, 101, 1583-1628.

5. J. Als-Nielsen and D. McMorrow, in Elements of Modern X-ray Physics, John Wiley \& Sons, Ltd, 2011, pp. 1-28.

6. R. Herbst-Irmer, J. Henn, J. J. Holstein, C. B. Hübschle, B. Dittrich, D. Stern, D. Kratzert and D. Stalke, The Journal of Physical Chemistry A, 2013, 117, 633-641.

7. S. Caticha-Ellis, Anomalous dispersion of x-rays in crystallography, University College Cardiff Press, Cardiff, Wales, 1981.

8. Bučinský, L., Jayatilaka, D., Grabowsky, S. (2016) The Journal of Physical Chemistry A, 120, 6650-6669.

9. Kleemiss, F.; Dolomanov, O. V.; Bodensteiner, M.; Peyerimhoff, N.; Midgley, L.; Bourhis, L. J.; Genoni, A.; Malaspina, L. A.; Jayatilaka, D.; Spencer, J. L.; White, F.; Grundkötter-Stock, B.; Steinhauer, S.; Lentz, D.; Puschmann, H.; Grabowsky, S. (2021) Chem. Sci., 12, 1675-1692.

Keywords: heavy atoms; charge density; quantum crystallography; relativistic effects

Support of this work by the National Science Centre, Poland through grant PRELUDIUM no. UMO-2018/31/N/ST4/02141 is gratefully acknowledged.

The experiment was carried out at the Spring-8 with the approval of the Japan Synchrotron Radiation Research Institute (Proposal Number 2019A1069). 